\title{
Metal-coated Glass Microfiber for Concentration Detection in Gas Mixtures using the 3- Omega Excitation Method
}

\author{
Sampath Kommandur, ${ }^{\mathrm{a}}$ Alireza Mahdavifar, ${ }^{\mathrm{a}}$ Shikai Jin, ${ }^{\mathrm{a}}$ Peter J. Hesketh, ${ }^{\mathrm{a}}$ and Shannon Yee ${ }^{\mathrm{a}+}$ \\ ${ }^{a}$ G.W.W. School of Mechanical Engineering, Georgia institute of Technology, \\ 771 Ferst Dr., Atlanta GA 30332, USA. \\ +Corresponding Authors: shannon.yee@me.gatech.edu
}

\begin{abstract}
The 3-Omega measurement technique was applied to a fabricated metal-coated glass fiber for the purpose of low power gas sensing. The sensor performance was evaluated for mixtures of $\mathrm{CO}_{2}, \mathrm{Ar}, \mathrm{He}$ and $\mathrm{CH}_{4}$ in $\mathrm{N}_{2}$ in an isothermal chamber, where mass flow controllers precisely controlled concentrations. The metal-coated fiber was fabricated by depositing a thin layer of gold $(\sim 150 \mathrm{~nm})$ onto a glass fiber, using a custom designed deposition lathe installed in a standard sputtering system. A custom 3-Omega conditioning circuit controls the AC heating current and detection of the 3-Omega voltage signal. The amplitude and phase lag, and the inphase and out-of-phase components of the 3-Omega voltage signal are presented for different gas mixtures and are related directly to their concentrations. Using this gas sensing technique, we have demonstrated the uncertainty in concentration (i.e., sensitivity) to be better than $50 \mathrm{ppm}$, and as low at $10 \mathrm{ppm}$ for some gases. The dependence of the different 3-Omega signals on the thermophysical properties of the system is briefly described. The low power, high sensitivity nature of the sensor is also demonstrated as the metal-coated fiber sensor consumes $\sim 10 \%$ of the power consumed by conventional thermal conductivity detectors (TCDs), and unlike those, it operates at near room temperatures.
\end{abstract}

\section{KEYWORDS}

3-Omega technique; Low power gas sensing; Thermal Conductivity Detector (TCD); Metalcoated glass fiber; AC excitation

\section{Introduction}

Gas sensors have been widely used to determine the composition of gas mixtures and to detect the presence of a particular species in gases. [1] Thermal conductivity detectors (TCDs) are a common type of gas sensors, which use the difference in thermal conductivity of gases to detect the composition of gas mixtures. Miniature TCDs are highly desirable for gas chromatography due to their excellent sensitivity demonstrated to $1 \mathrm{ppm}$. Nanoscale bridge type sensors using nanotubes [2], nanowires [3], and nanobelts [4] have also been fabricated. These TCDs often require high power consumption (hundreds of milliWatts to a few Watts) to provide sensitivities in the few ppm range. Smaller variation of microTCDs are fabricated that offer better sensitivity and power consumption [5]. However some of these TCDs operate at high temperatures [6], which necessitate routine re-calibration and may be destructive to the detected 
sample. More recently, we have demonstrated the ability to perform low power gas sensing using the 3-Omega technique on a polysilicon microbridge geometry while still maintaining good sensitivity [7]. However, in the microbridge design a significant portion of the heat was conducted along the microbridge to the substrate, which reduced the heat input to the surrounding gas medium, thereby limiting the sensitivity of 3-Omega technique to $1,000 \mathrm{ppm}$. Herein, we present a gas sensing technique that overcomes not only these challenges, but offers other advantages such as ease of fabrication, near room temperature measurements while still consuming power in the order of milliWatts. The design and fabrication technique of the sensor used in this work was first developed by Schiffres et al [8]. for the measurement of thermal conductivity of liquids and gases.

\subsection{Principles of 3-Omega}

The 3-Omega technique has been extensively used in thermal conductivity measurements [9] and is well described in literature [10], but it also offers an approach to low-power gas sensing that we have recently investigated. [7] In a standard 3-Omega measurement, a sinusoidal electrical current of amplitude $I_{\omega}$ at frequency $\omega=2 \pi f$ is driven through a metal heater line, resulting in a Joule heating at frequency $2 \omega$. The periodic heating creates a thermal wave that penetrates the surrounding medium. The thermal wave attenuates over a penetration depth, given by $L_{p}=\sqrt{\alpha / 2 \omega}$, where $\alpha$ is the thermal diffusivity of the surrounding medium. This gives rise to a temperature oscillation at the source, which has a frequency $2 \omega$ but lags the heating current by a phase lag $\varphi$ due to the finite time it takes for a temperature response. The temperature oscillation causes the resistance of the heater to oscillate at $2 \omega$. Since the current is driven at $1 \omega$, this resistance oscillation results in a voltage component at $3 \omega$. The amplitude, $V_{3 \omega}$ and phase lag, $\varphi$ of the voltage signal can be directly measured and the voltage amplitude can be directly related to the amplitude of temperature oscillation by Eq. (1).

$$
\Delta T_{2 \omega}=\frac{2}{d R / d T} \cdot \frac{V_{3 \omega, \mathrm{RMS}}}{I_{\omega, \mathrm{RMS}}}
$$

where $d R / d T$ is the temperature rate of change of the sensor's resistance and the subscript RMS refers to the root-mean-square value.

The amplitude and phase signals can be directly related to the thermal properties (e.g., thermal diffusivity) of the sensor and the gas medium. Since the thermal diffusivity is a function of the gas mixture, the 3-Omega signals vary with gas composition and can be used to resolve gas compositions. The phase lag is only a function of the geometry and thermophysical properties of the heater and its environment, and is independent of the amplitude of driving current thus uniquely alleviating the need for routine calibration.

\section{The 3-Omega sensor}

In our previous study on 3-Omega gas sensing, a polysilicon microbridge suspended on a silicon dioxide sacrificial layer was used which could readily resolve gas concentrations of 1,000 
ppm in binary mixtures [7]. Since a significant portion of the heat was conducted within the microbridge and dissipated to the solid substrate, the sensitivity to the gas medium was limited. In this study, a glass fiber coated with a thin layer of metal (gold) is used as the 3-Omega heater and the sensing element. The raw glass fiber has a $26 \mu \mathrm{m}$ diameter, and consists of a leaded glass core (Schott F2) and a borosilicate cladding (Schott 8250) [8]. The diameters and properties of the different layers are provided in Table 1.

\subsection{Fabrication of sensor}

The metal layer was deposited using a conventional sputtering system (Unifilm Multisource Sputtering System, IEN, Georgia Tech). To achieve uniform cylindrical coating, the fibers were strung tautly onto a spool, which was uniformly rotated with the motor in the sputtering system as shown in Fig. 1[8]. The deposition lathe ensured uniform circumferential coating. Since the deposition crystal monitor is calibrated for deposition in a rectangular plane, the thickness deposited on the sensors is smaller by a factor of $\pi$, which results from the ratio of the fiber's actual surface area $(\pi \times$ diameter $\times$ length) to its projected area (diameter $\times$ length). The sputterer was programmed to deposit a $10 \mathrm{~nm}$ titanium adhesion layer, followed by a 150 $\mathrm{nm}$ of gold. The fiber was characterized using a SEM to determine the exact thickness and to verify the uniformity of the gold layer along the circumference of the fiber. This was done by measuring the metal layer's thickness at over 10 points along the fiber's circumference. The resulting thickness of the metal layer was $135 \pm 11 \mathrm{~nm}$ (see Supplemental Information for more details). Figure 2 shows an SEM image of the fiber's cross section. Figure 3 shows a picture of the metal-coated sensor along with a schematic highlighting the electrical connections and a cross-section of the fiber.

\subsection{Experimental set-up}

The gold-coated fiber was placed in a dual-in line package (DIP) and wired in a fourpoint configuration. Electrical contacts to the fiber were made using DuPont $\subset$ CB028 silver conductive paste. The sensor was placed in an isothermal chamber whose temperature was uniformly increased from $20{ }^{\circ} \mathrm{C}$ to $50{ }^{\circ} \mathrm{C}$ in $5{ }^{\circ} \mathrm{C}$ increments and the resistance's temperature dependence $d R / d T$ was measured to be $0.1155 \pm 0.008 \mathrm{ohm} /{ }^{\circ} \mathrm{C}$ (see Supplemental Information for details). An experimental set-up similar to the one described in our previous work was used [7]. An input current amplitude of $12 \mathrm{~mA}$ was used and frequencies were swept between 0.5 and $2000 \mathrm{~Hz}$. 3-Omega measurements were carried out on mixtures of trace gases ( $\mathrm{He}, \mathrm{Ar}, \mathrm{CO}_{2}$ and $\mathrm{CH}_{4}$ ) in $\mathrm{N}_{2}$. The concentration of the trace gas was varied between 0 and $5 \%$ in increments of $0.5 \%$. At each concentration, the amplitude $(\Delta T)$, phase lag $(\phi)$, and the in-phase $(X)$ and out-ofphase $(Y$ ) components of $\Delta T$ from the SR850 lock-in amplifier were recorded (see Eq. (2) for analytical relationships). The measurements were repeated multiple times for each concentration and the resulting standard deviation was used to estimate the experimental uncertainty.

$$
X=\Delta T \cos \phi \quad Y=\Delta T \sin \phi
$$




\section{Results and discussion}

\subsection{Raw signals}

Figure 4 shows an example raw output signal of the $3 \omega$ measurements on the sensor in He- $\mathrm{N}_{2}$ mixture for a current amplitude of $12 \mathrm{~mA}$. Similar data and trends were observed for other gas mixtures (Ar, $\mathrm{CH}_{4}$ and $\mathrm{CO}_{2}$ in $\mathrm{N}_{2}$, see Supplemental Information for data). The plots show the frequency dependence of (a) amplitude of temperature oscillation $\Delta T$, (b) phase lag, as well as the (c) in-phase $(X)$ and (d) out-of-phase $(Y)$ parts of $\Delta T$, respectively. As discussed in our previous work, the system can be approximated to be in quasi-steady state at lower frequencies with a large penetration depth, where the thermal transport is primarily governed by the thermal conductivity of the gas mixture. Table 2 summarizes the relevant thermophysical properties of the five gases used at room temperature [11]. Since He has a higher thermal conductivity than $\mathrm{N}_{2}$, a system with higher concentration of $\mathrm{He}$ in $\mathrm{N}_{2}$ dissipates heat better. This leads to a decrease in the amplitude of temperature oscillation as the concentration of He increases. The phase lag, however is most sensitive to gas concentration at frequencies near $600 \mathrm{~Hz}$, which is the characteristic frequency of the sensor-gas system and also exhibits a maximum at this frequency. As discussed in our previous work [7], this characteristic frequency is related to the geometry and the thermophysical properties of the sensor-gas system. The phase lag describes the thermal response of the system and thus, is dictated by the specific heat of the gas mixture. Since He has a higher specific heat than $\mathrm{N}_{2}$, a system with higher concentration of $\mathrm{He}$ in $\mathrm{N}_{2}$ responds slower. This leads to an increase in phase lag as the concentration of He increases. The curves for $X$ and $Y$ exhibit similar trends to that of the amplitude, where the maximum sensitivity to gas composition is observed at low frequencies.

\subsection{Composition curves}

Based on the raw 3-Omega signals, composition curves are developed which can be used to determine the composition of an unknown mixture using the measured signal. These curves present the difference in the 3-Omega signal between the gas mixture and pure $\mathrm{N}_{2}$ reference gas as a function of the trace gas concentration. Figure 5 shows the difference in 3-Omega signals $\left(\Delta T, \phi, X\right.$ and $Y$ ) between the He- $\mathrm{N}_{2}$ mixture and pure $\mathrm{N}_{2}$ as a function of frequency for different concentrations of He. Similar curves were obtained for the other mixtures as well (see Supplemental Information). As mentioned before, $\Delta T, X$ and $Y$ are most sensitive to concentration at low frequencies while phase lag exhibits better sensitivity near the characteristic frequency. To better study the effect of mixture composition on this differential 3-Omega signal, we consider the differential $\Delta T, X$ and $Y$ between the frequencies 3 and $10 \mathrm{~Hz}$ to focus on the range of most sensitivity. These regions of maximum sensitivity are highlighted in the Fig. 5. We use the average over these 10 points instead of a single point (frequency) to smooth over (noise) fluctuations. These average values are then plotted against mixture concentration for all gas mixtures to obtain composition curves based on $\Delta T, X$ and $Y$, which are depicted in Figs. $6(\mathrm{a}),(\mathrm{c})$ and (d), respectively. A linear fit between the differential signal and concentration was 
performed and serve as the composition curves for the sensor. A similar analysis was carried out on the phase lag where the frequency range between $200-900 \mathrm{~Hz}$ was used; this range contains the characteristic frequency and exhibits the highest sensitivity. The corresponding composition curve is shown in Fig. 6(b). Using these composition curves, it is possible to determine the composition of an unknown binary mixture based on the differential $3 \omega$ signal.

To determine the sensitivity of the sensor, an uncertainty analysis was carried out on the composition curves. Eq. (3) can be used to calculate the uncertainty in concentration, where $\delta($ signal) is the uncertainty in the measured $3 \omega$ signal and $m$ is the slope of the composition curve based on the corresponding signal. The uncertainty of the measured signals was calculated from the standard deviation between multiple $3 \omega$ measurements using Student's t-distribution. The composition curve is highly linear $\left(R^{2}=0.99\right)$ and the slope is well characterized $(m=0.143 \pm 0.003$, from $95 \%$ confidence interval), therefore uncertainty in the slope does not significantly add appreciable uncertainty to the composition uncertainty. The analysis is similar to the one presented in our previous work [7]. Table 3 lists the uncertainty in the concentration for the different gas mixtures. As can be seen from Table 3, the composition curve based on $\Delta T$ generally provides the best sensitivity in concentration. The composition curve based on $\phi$, while it has the worst sensitivity remains unchanged regardless of the input current. This is beneficial because it removes the need for routine sensor calibration.

$$
\delta(\%)=\left|\frac{\sqrt{2} \cdot \delta(\text { signal })}{m}\right|
$$

It is also possible to determine the minimum detectable concentration of the gases based on the composition curves using Eq. (4), where $c$ is the y-intercept of the composition curve. Table 4 summarizes this minimum concentration for different gas mixtures, based on each of the four $3 \omega$ signals.

$$
\operatorname{Min} \%=\delta(\%)+\left|\frac{c}{m}\right|
$$

As can be observed, it is possible to detect gases with uncertainties in measurement (i.e., sensitivities) lower than $10 \mathrm{ppm}$ for gas mixtures of components with thermophysical properties differing by an order of magnitude (e.g., He and $\mathrm{N}_{2}$ ). For mixtures of components with similar properties (e.g., $\mathrm{CH}_{4}$ and $\mathrm{N}_{2}$ ), the sensitivity is $\sim 50 \mathrm{ppm}$; improved sensitivity to this gas can be achieved by using a different reference gas when properties are similar (e.g., use He instead of $\mathrm{N}_{2}$ when measuring $\mathrm{CH}_{4}$ ).

\subsection{Thermal Conductivity Sensing}

Schiffres et al. [8] developed a generalized $n$-layer concentric cylindrical periodic heating solution and applied it to determine the thermal conductivity of gases using a similar metalcoated fiber heater approach; caution needs to be used when applying the mathematic model presented by Schiffres et al. as a few minor mathematic errors are present in the publication (see Supplemental Information corrections). The amplitude data $(\Delta T)$ was fit to the model to 
determine the thermal conductivity of our mixture for different gases and concentrations. Figure 7 shows the thermal conductivity as a function of the concentration of the trace gas in the mixture. These curves provide another way to quantify an unknown mixture based on its thermal conductivity, which does not require comparison to the $3 \omega$ signals of $\mathrm{N}_{2}$. However, it must be noted that the resolution based on the thermal conductivity sensing is significantly worse than that based on the other composition curves since this is based on a fitted model propagating error and not the direct 3-Omega signals themselves.

\section{CONCLUSIONS}

In this work, the 3-Omega technique was used on a metal-coated microfiber for low power, high resolution gas sensing at near room operational temperatures. The sensor was fabricated by sputtering on a custom deposition lathe. The phase lag signal exhibits a maximum at a frequency corresponding to the characteristic frequency of the system, where it exhibits the maximum sensitivity as well. Composition curves were developed for different gas mixtures based on each of the four output signals of the 3-Omega measurement. The composition curve based on phase lag is independent of the driving current, which eliminates the need for individual sensor calibration. Herein, we have shown that it is possible to determine gas concentrations with a resolution as small as $10 \mathrm{ppm}$. This sensitivity was achieved with a RMS power consumption of $\sim 5 \mathrm{~mW}$ (determined using $P_{\mathrm{RMS}}=I_{\mathrm{RMS}}{ }^{2} \mathrm{R}$ ). This work offers the advantage of sensitivities that are comparable to some of the best commercial sensors, while consuming power that is two orders of magnitude lower. Furthermore, the maximum temperature reached by the sensor, which is the sum of the DC and maximum AC temperature rise during operation is $\sim 25^{\circ} \mathrm{C}$. The $\mathrm{AC}$ temperature rise is directly related to the 3-Omega signal whereas the DC temperature rise is obtained indirectly from the change in resistance between zero current and a current of $12 \mathrm{~mA}$, and the sensor's $\mathrm{dR} / \mathrm{dT}$. This temperature rise is significantly less than other thermal conductivity gas sensors that require elevated temperatures $\left(\sim 100{ }^{\circ} \mathrm{C}\right)$ for high sensitivities. This results in improved sensor lifetime and reduces the frequency of calibration. Furthermore, the fabrication of the metal-coated fiber is simpler than microfabricated bridges, as it does not require photolithography. Finally, since the amplitude and phase lag are independent and governed by different thermal properties (e.g., thermal conductivity and specific heat), it is now possible for this technique to be extended to ternary gas mixtures.

\section{ACKNOWLEDGEMENTS}

The authors would like to thank Adis Halilovic, Abbas Furniturewala, and our colleague Alexander Limia for helping with the design of the deposition lathe. This work was performed in part at the Georgia Tech Institute for Electronics and Nanotechnology, a member of the National nanotechnology Coordinated Infrastructure, which is supported by the National Science Foundation (Grant ECCS-1542174).

\section{REFERENCES}


[1] A. Azad, S. Akbar, S. Mhaisalkar, L. Birkefeld, K. Goto, Solid-state gas sensors: A review, Journal of the Electrochemical Society, 139(1992) 3690-704.

[2] T. Kawano, H.C. Chiamori, M. Suter, Q. Zhou, B.D. Sosnowchik, L. Lin, An electrothermal carbon nanotube gas sensor, Nano letters, 7(2007) 3686-90.

[3] M.W. Ahn, K.S. Park, J.H. Heo, D.W. Kim, K.J. Choi, J.G. Park, On-chip fabrication of ZnO-nanowire gas sensor with high gas sensitivity, Sensors and Actuators B: Chemical, 138(2009) 168-73.

[4] C. Yu, Q. Hao, S. Saha, L. Shi, X. Kong, Z.L. Wang, Integration of metal oxide nanobelts with microsystems for nerve agent detection, Applied Physics Letters, 86(2005) 063101--3.

[5] A. Mahdavifar, R.J. Aguilar, Z. Peng, P.J. Hesketh, M. Findlay, J.R. Stetter, et al., Simulation and fabrication of an ultra-low power miniature microbridge thermal conductivity gas sensor, Journal of Electrochemical Society, 161(2014) 855-61.

[6] D. Cruz, J. Chang, S. Showalter, F. Gelbard, R. Manginell, M. Blain, Microfabricated thermal conductivity detector for the micro-ChemLab ${ }^{\mathrm{TM}}$, Sensors and Actuators B: Chemical, 121(2007) 414-22. [7] S. Kommandur, A. Mahdavifar, P.J. Hesketh, S. Yee, A microbridge heater for low power gas sensing based on the 3-Omega technique, Sensors and Actuators A: Physical, 233(2015) 231-8.

[8] S.N. Schiffres, J.A. Malen, Improved 3-omega measurement of thermal conductivity in liquid, gases, and powders using a metal-coated optical fiber, The Review of scientific instruments, 82(2011) 064903. [9] D.G. Cahill, Thermal conductivity measurement from 30 to $750 \mathrm{~K}$ : the $3 \omega$ method, Review of Scientific Instruments, 61(1990) 802-8.

[10] C. Dames, G. Chen, $1 \omega, 2 \omega$, and $3 \omega$ methods for measurements of thermal properties, Review of Scientific Instruments, 76(2005) 124902.

[11] P.J. Linstrom, W.G. Mallard, Eds., NIST Chemistry WebBook, NIST Standard Reference Database Number 69, National Institute of Standards and Technology, Gaithersburg MD, 20899.

[12] T.L. Bergman, A.S. Lavine, F.P. Incropera, D.P. DeWitt, Fundamentals of Heat and Mass Transfer, 7th ed., (2011) 983-6. 


\begin{tabular}{|c|c|c|c|c|}
\hline & $\begin{array}{c}\text { Layer 1 } \\
\text { Schott F2 }^{\mathbf{a}} \\
\text { leaded glass) }\end{array}$ & $\begin{array}{c}\text { Layer 2 } \\
\text { (Schott 8250 } \\
\text { borosilicate) }\end{array}$ & $\begin{array}{c}\text { Layer 3 } \\
\text { (Titanium) }\end{array}$ & $\begin{array}{c}\text { Layer 4 } \\
\text { (Gold) }\end{array}$ \\
\hline $\begin{array}{c}\text { Diameter (d)/ } \\
\text { Thickness (t) }\end{array}$ & $\mathrm{d}=24 \mu \mathrm{m}$ & $\mathrm{t}=1.1 \mu \mathrm{m}$ & $\mathrm{t}=10 \mathrm{~nm}$ & $\mathrm{t}=150 \mathrm{~nm}$ \\
\hline $\begin{array}{c}\text { Thermal Conductivity } \\
(\mathbf{W} / \mathbf{m}-\mathbf{K})\end{array}$ & 0.78 & 1.18 & 21.9 & 317 \\
\hline $\begin{array}{c}\left.\text { Density (kg/m } \mathbf{~}^{3}\right) \\
\text { Specific Heat (J/kg-K) }\end{array}$ & 3600 & 2280 & 4500 & 19300 \\
\hline
\end{tabular}

a Properties and dimensions were supplied by the manufacturer

Table 1: Dimensions and properties of the different layers of the metal-coater fiber sensor[8]. The values for the fiber layers were provided by the manufacturer and the properties of the titanium and gold layers are obtained from Ref. [11]. 


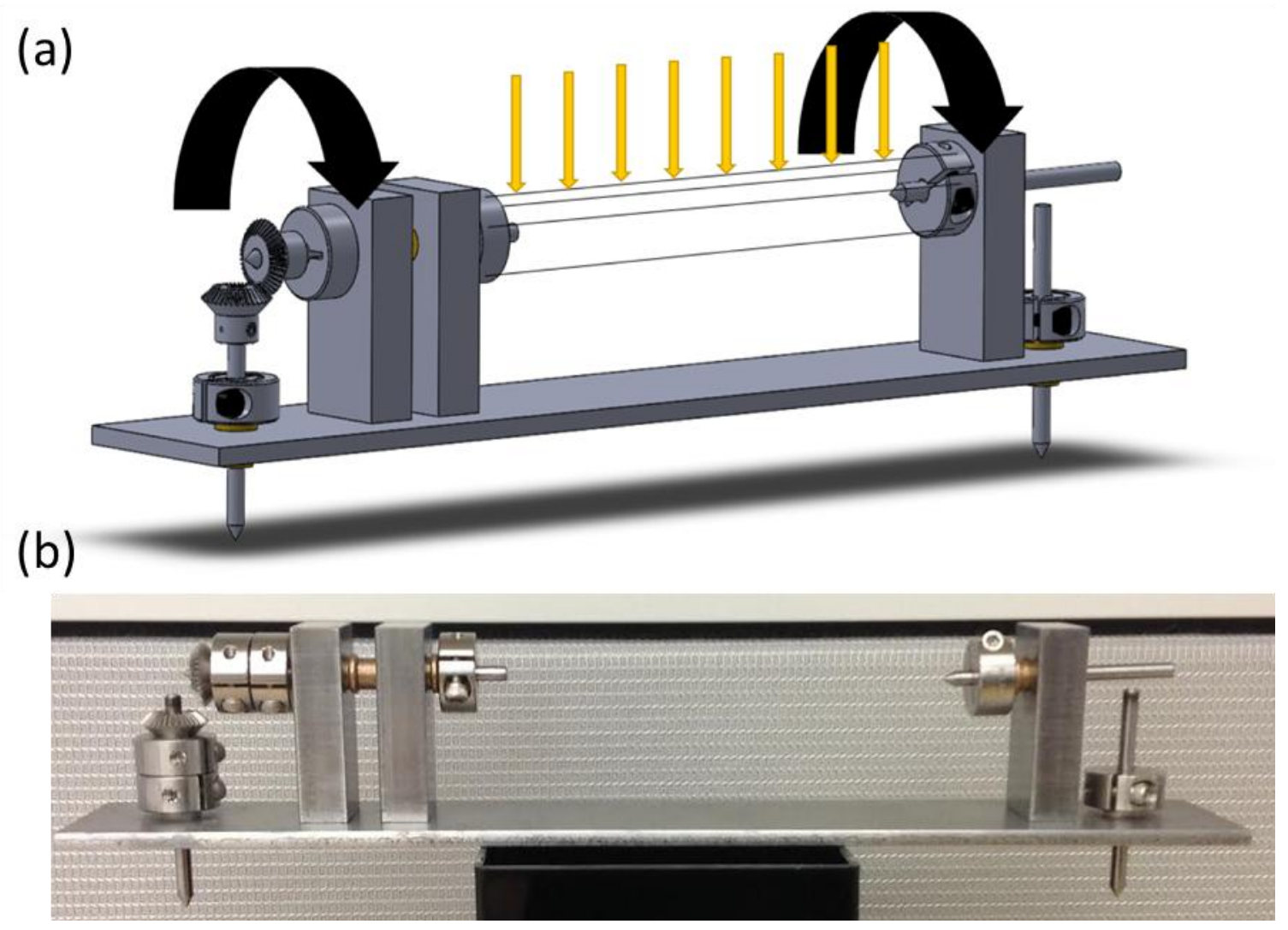

Figure 1: (a) Schematic showing the deposition lathe with fibers strung onto the rotating spool. The black arrows indicate the direction of rotation of the spool and the golden arrows indicate the direction of gold sputtering. A bevel gear is used to convert the plane of rotation from the default horizontal plane to the vertical one with the spool. (b) A picture of the deposition lathe used. 

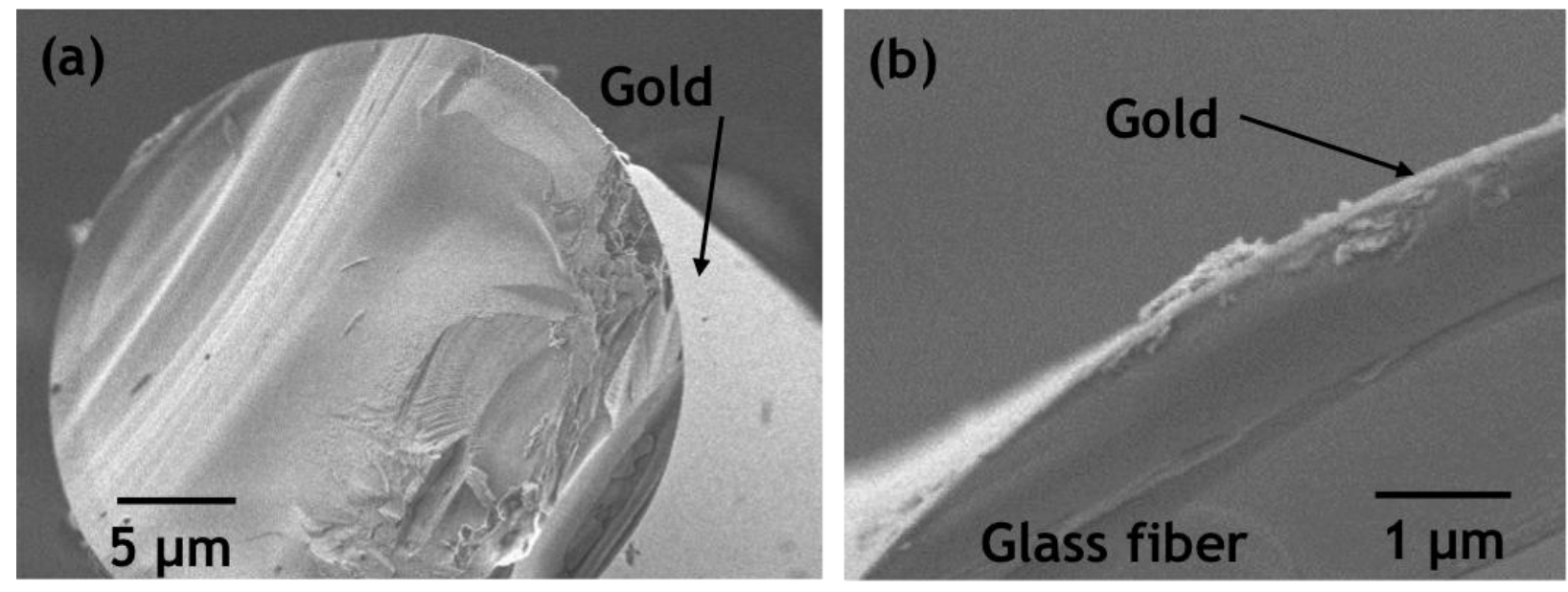

Figure 2: (a) The cross-sectional image of the metal-coated fiber highlighting the gold layer. (b) A magnified image of the gold layer on the fiber. The uniformity of the coating was verified by measuring the thickness of the gold at several points along the circumference. An Energy Dispersive X-Ray Spectroscopy (EDS) study was also carried out on the fiber to verify the gold thickness. 


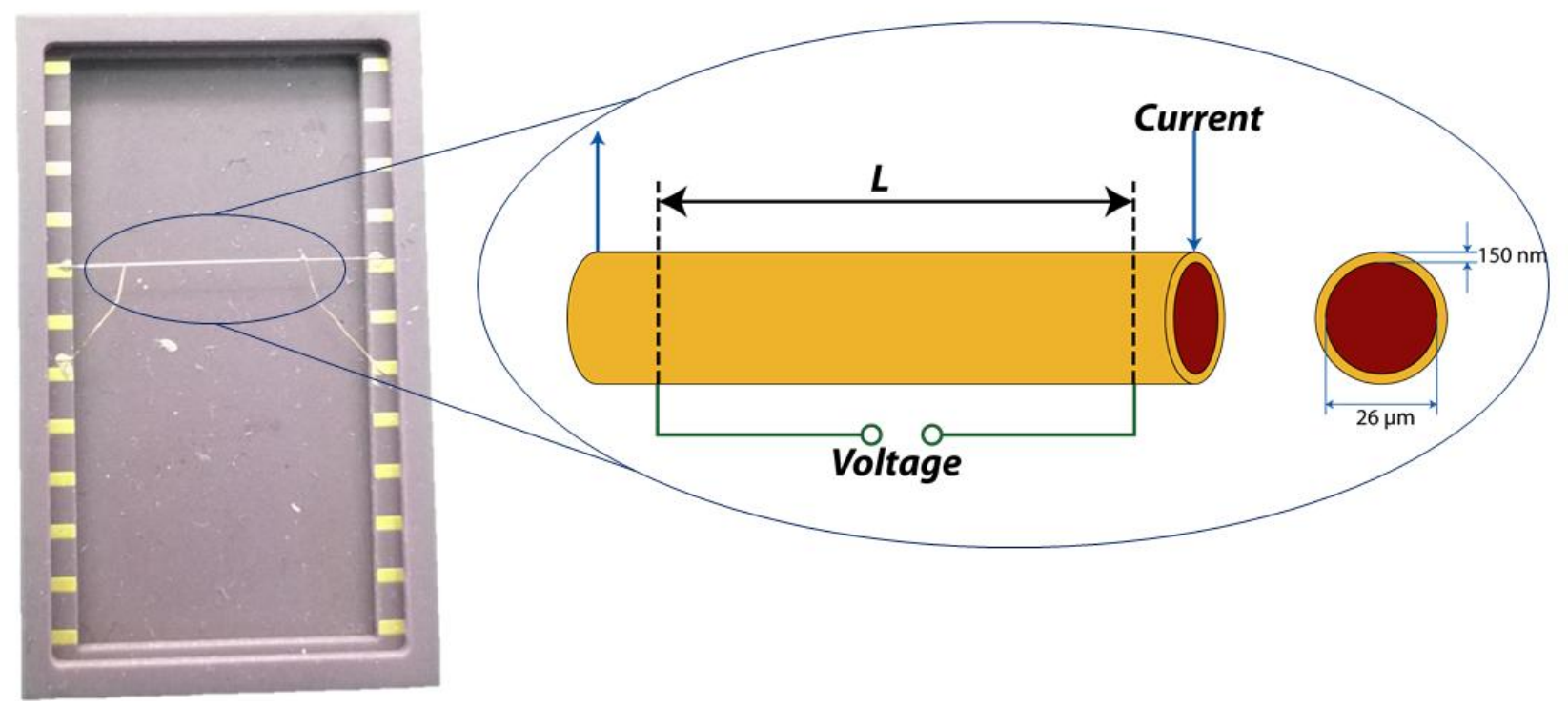

Figure 3: A picture of the metal-coated fiber sensor with its electrical connections along with a schematic. Also shown is a cross-sectional schematic of the fiber indicating the dimensions of the fiber and the metal layer. 
(a)

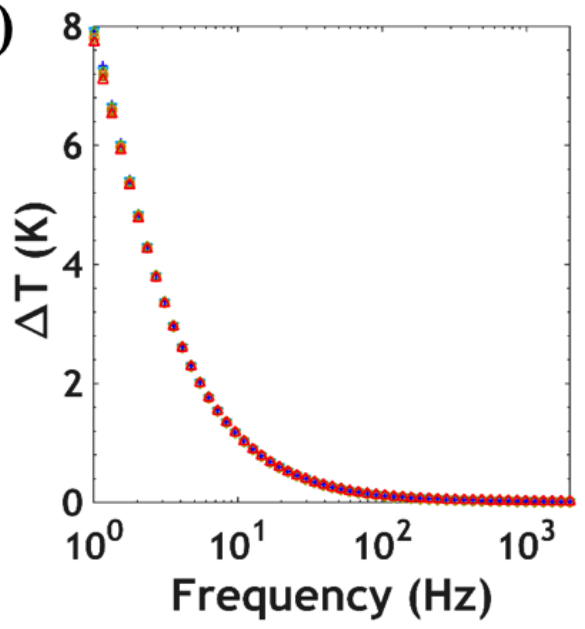

(c)

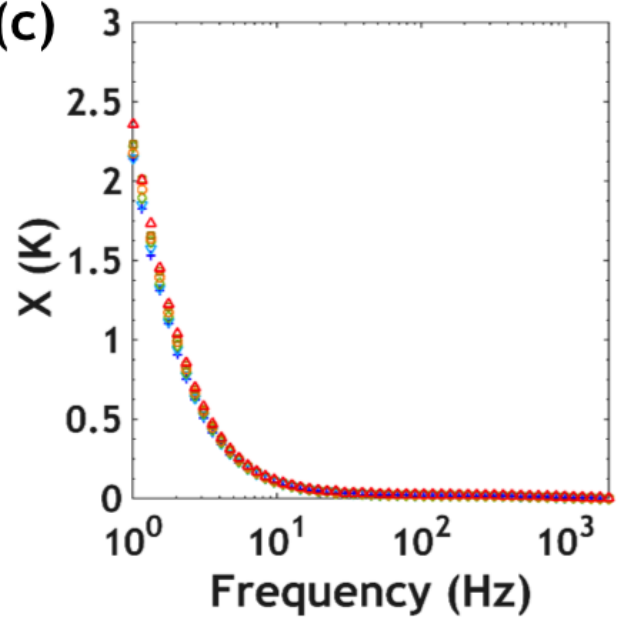

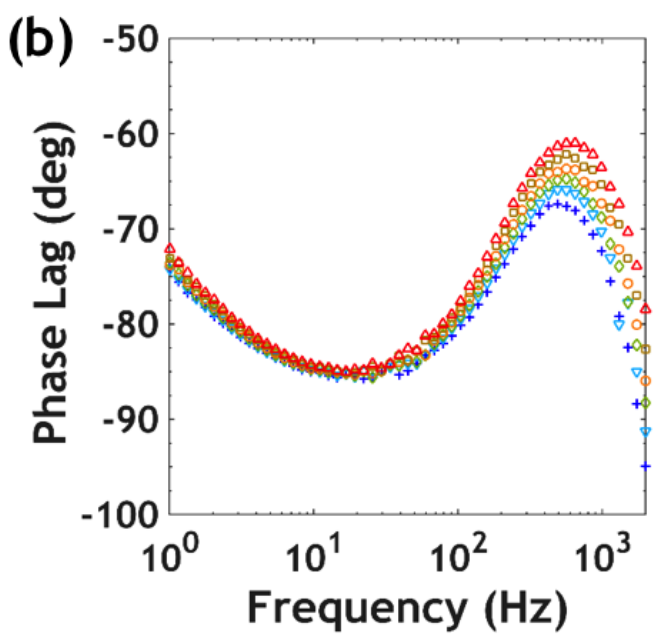

(d)

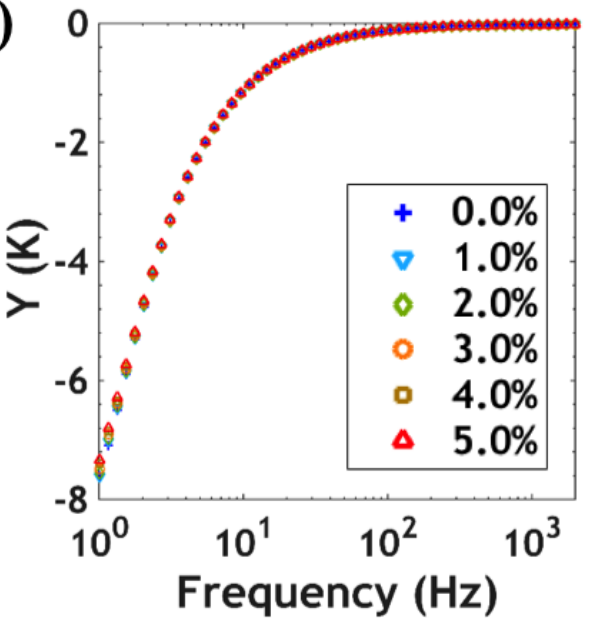

Figure 4: The raw 3-Omega signals for the $\mathrm{He}-\mathrm{N}_{2}$ mixture, for six different compositions, and a current amplitude of $12 \mathrm{~mA}$. Figures (a) and (b) indicate the amplitude and phase lag, while (c) and (d) indicate the in-phase (real) and out-of-phase (imaginary) components of the amplitude, respectively. The amplitude, $\mathrm{X}$ and $\mathrm{Y}$ are most sensitive at low frequencies whereas the phase lag is most sensitive at a frequency close to the characteristic frequency of the system. The phase lag also exhibits a maximum at a characteristic frequency of the system. 


\begin{tabular}{|c|c|c|c|c|}
\hline Material & $\begin{array}{c}\text { Thermal } \\
\text { Conductivity } \\
(\mathbf{W} / \mathbf{m}-\mathbf{K})\end{array}$ & $\begin{array}{c}\text { Density } \\
(\mathbf{k g} / \mathbf{m})\end{array}$ & $\begin{array}{c}\text { Heat } \\
\text { Capacity } \\
(\mathbf{J} / \mathbf{k g}-\mathbf{K})\end{array}$ & $\begin{array}{c}\text { Thermal } \\
\text { Diffusivity } \\
(\mathbf{m} / \mathbf{s})\end{array}$ \\
\hline Nitrogen & 0.0258 & 1.123 & 1041.8 & $2.21 \times 10^{-5}$ \\
\hline Methane & 0.0346 & 0.644 & 2235.8 & $2.40 \times 10^{-5}$ \\
\hline Helium & 0.1560 & 0.160 & 5193.0 & $1.87 \times 10^{-4}$ \\
\hline Argon & 0.0177 & 1.603 & 521.5 & $2.12 \times 10^{-5}$ \\
\hline $\begin{array}{c}\text { Carbon } \\
\text { dioxide }\end{array}$ & 0.0168 & 1.773 & 852.5 & $1.11 \times 10^{-5}$ \\
\hline
\end{tabular}

Table 2: Relevant thermophysical properties of the different gases used in this work. [9] 

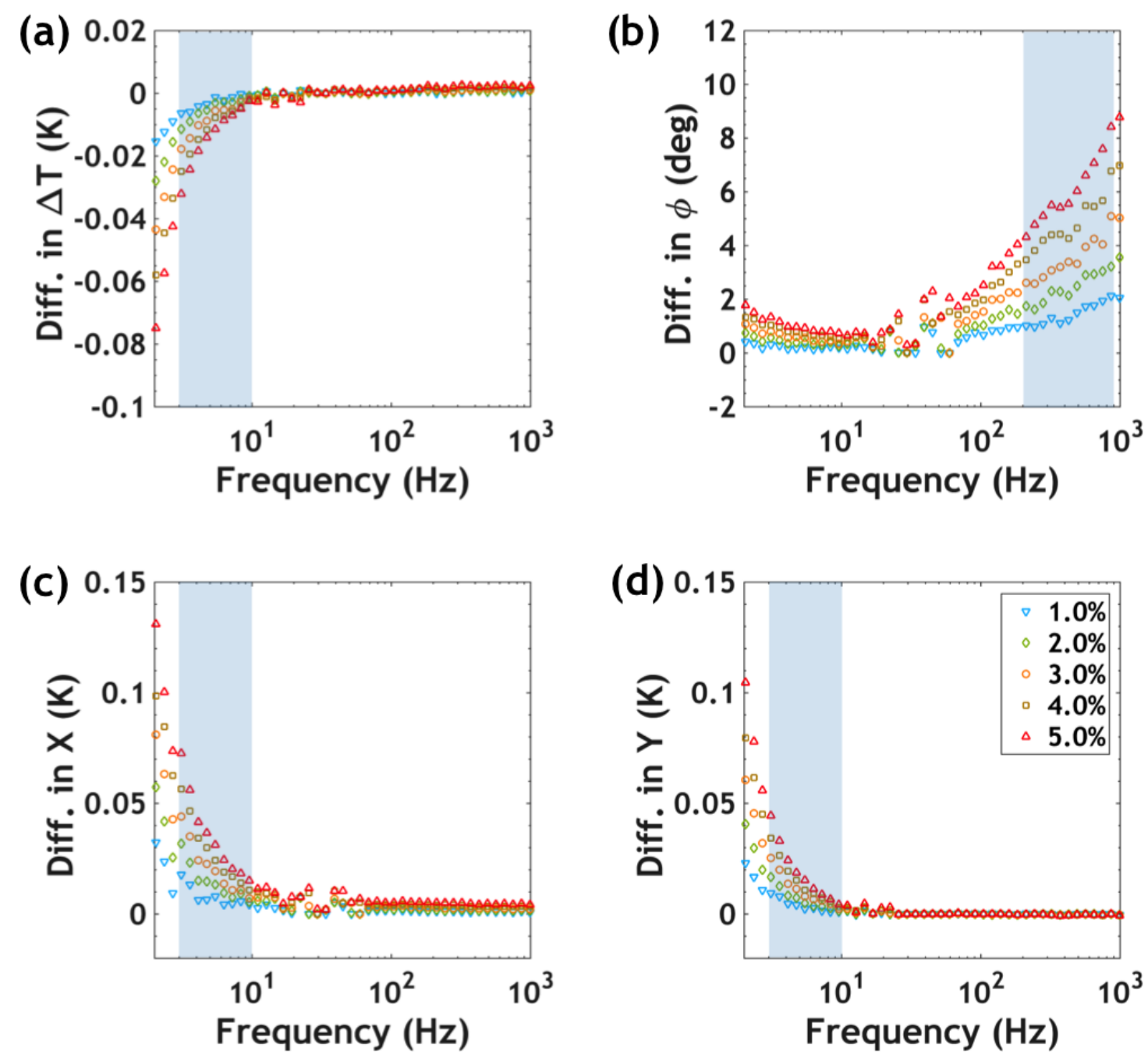

Figure 5: Differential signals of amplitude, phase, $X$ and $Y$ vs. frequency for different compositions of $\mathrm{He}$ in $\mathrm{N}_{2}$. The sensitivity of the different signals at different frequencies can be readily noticed. The frequency range of high sensitivity considered for the composition curves is highlighted in each of the four figures. 
(a)

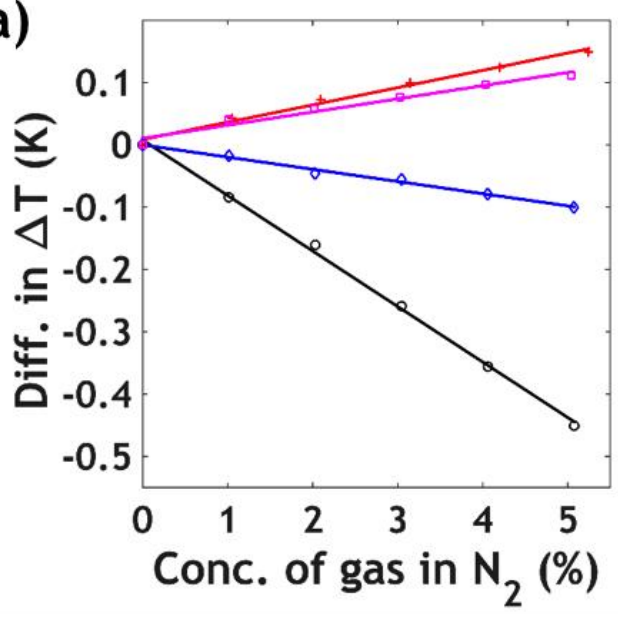

(c)

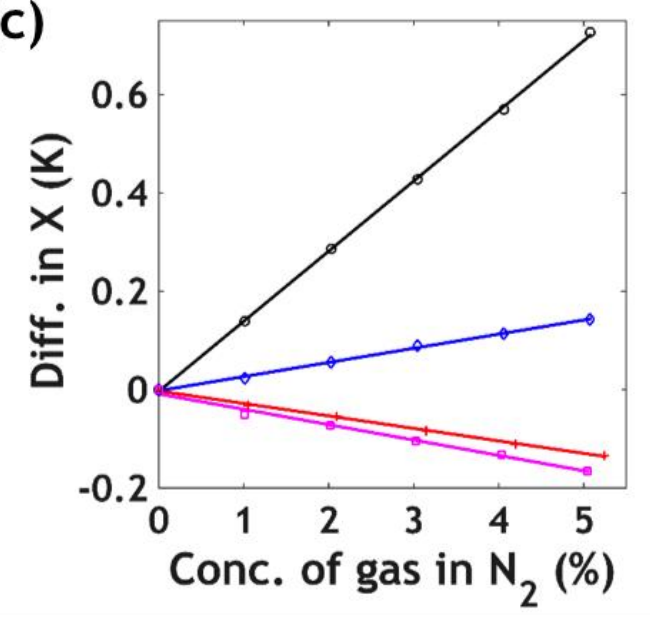

(b)

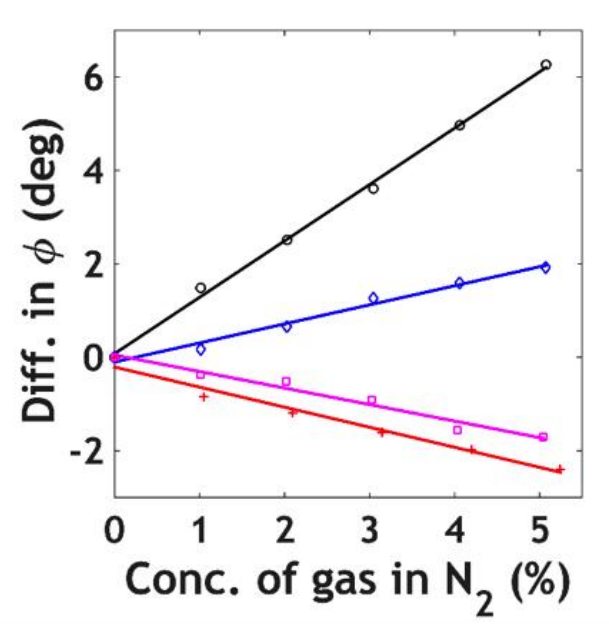

(d)

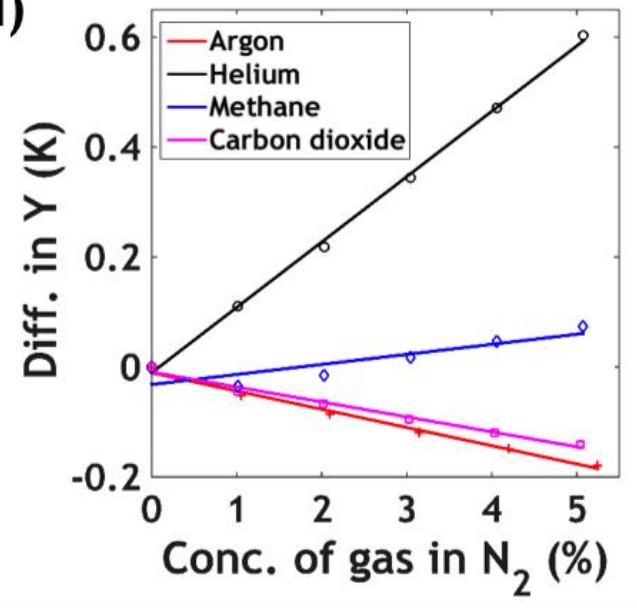

Figure 6: Composition curves for all four gas mixtures based on differential 3-Omega signals. A linear trend between the averaged differential signal and concentration is observed for all cases, as indicated by a linear fit for the data. The composition curve based on phase lag is independent of driving current amplitude and eliminates the need for calibration for individual experiments. 


\begin{tabular}{|c|c|c|c|c|}
\hline \multirow{2}{*}{$\begin{array}{c}\text { Gas } \\
\text { Mixture }\end{array}$} & \multicolumn{4}{|c|}{$\begin{array}{c}\text { Uncertainty in measured } \\
\text { concentration using }\end{array}$} \\
\cline { 2 - 5 } & $\begin{array}{c}\boldsymbol{\phi} \\
\mathbf{p p m})\end{array}$ & $\begin{array}{c}\boldsymbol{X} \\
(\mathbf{p p m})\end{array}$ & $\begin{array}{c}\boldsymbol{Y} \\
(\mathbf{p p m})\end{array}$ \\
\hline Ar in $\mathbf{N}_{\mathbf{2}}$ & 18.7 & 916.4 & 43.3 & 22.9 \\
\hline $\mathbf{H e}$ in $\mathbf{N}_{\mathbf{2}}$ & 8.1 & 801.0 & 6.8 & 8.5 \\
\hline $\mathbf{C H}_{\mathbf{4}}$ in $\mathbf{N}_{\mathbf{2}}$ & 29.9 & 756.1 & 39.0 & 47.0 \\
\hline $\mathbf{C O}_{\mathbf{2}}$ in $\mathbf{N}_{\mathbf{2}}$ & 35.6 & 2400 & 32.7 & 37.9 \\
\hline
\end{tabular}

Table 3: Uncertainties in the measured concentration (i.e., sensitivity) of different gases for different composition curves 


\begin{tabular}{|c|c|c|c|c|}
\hline \multirow{2}{*}{ Gas Mixture } & \multicolumn{4}{|c|}{ Minimum detectable concentration using } \\
\cline { 2 - 5 } & $\boldsymbol{\Delta T}(\boldsymbol{\%})$ & $\boldsymbol{\phi}(\boldsymbol{\%})$ & $\boldsymbol{X}(\boldsymbol{\%})$ & $\boldsymbol{Y}(\boldsymbol{\%})$ \\
\hline Ar in $\mathbf{~}_{\mathbf{2}}$ & 0.289 & 0.527 & 0.0927 & 0.281 \\
\hline $\mathbf{H e}$ in $\mathbf{N}_{\mathbf{2}}$ & 0.0869 & 0.145 & 0.0449 & 0.0846 \\
\hline $\mathbf{C H}_{\mathbf{4}}$ in $\mathbf{N}_{\mathbf{2}}$ & 0.0556 & 0.310 & 0.556 & 0.684 \\
\hline $\mathbf{C O 2}$ in $\mathbf{N}_{\mathbf{2}}$ & 0.497 & 0.351 & 0.271 & 0.324 \\
\hline
\end{tabular}

Table 4: Minimum concentration of different gases that can be detected based on the composition curve used 


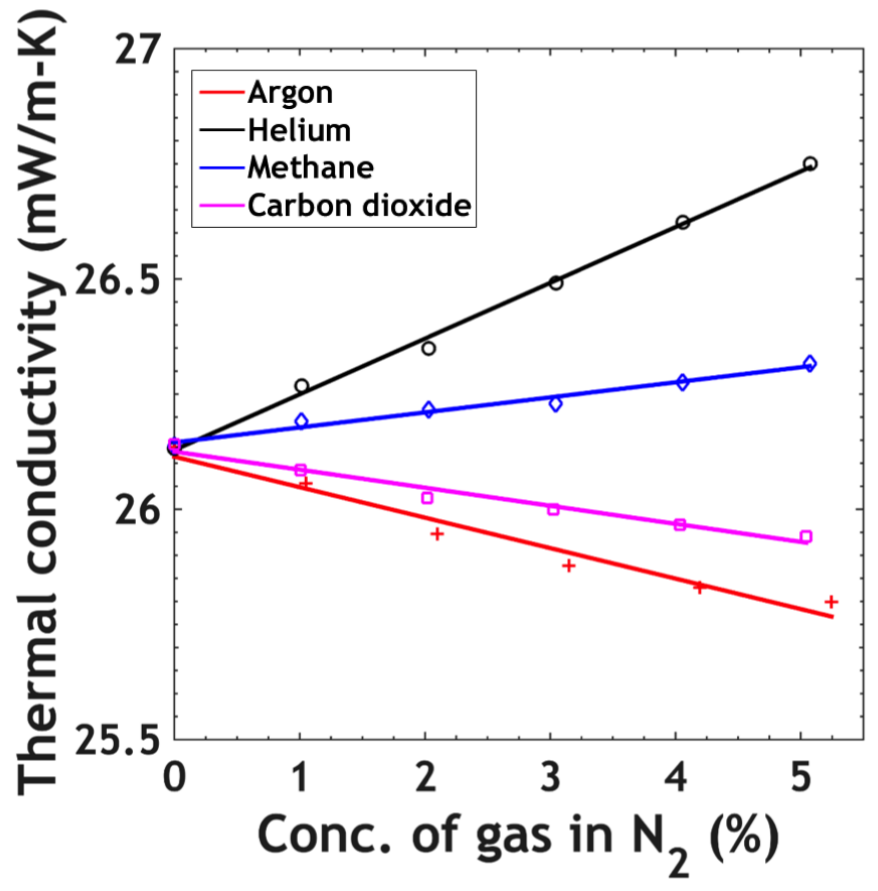

Figure 7: Thermal conductivity of different gas mixtures obtained by fitting the measured amplitude data to a standard heat transfer model for our sensor-gas system. This composition curve allows for determination of the concentration without relying on measurements of base gas. 